PRAMANA

- journal of

(c) Indian Academy of Sciences

Vol. 76 , No. 1

physics

January 2011

pp. 1-22

\title{
Results from PAMELA, ATIC and FERMI: Pulsars or dark matter?
}

\author{
DEBTOSH CHOWDHURY ${ }^{1, *}$, SUDHIR K VEMPATI $^{1}$ and \\ CHANDA J JOG ${ }^{2}$ \\ ${ }^{1}$ Centre for High Energy Physics, Indian Institute of Science, Bangalore 560 012, India \\ ${ }^{2}$ Department of Physics, Indian Institute of Science, Bangalore 560 012, India \\ *Corresponding author. E-mail: debtosh@cts.iisc.ernet.in
}

MS received 28 March 2010; revised 26 October 2010; accepted 27 October 2010

\begin{abstract}
It is well known that dark matter dominates the dynamics of galaxies and clusters of galaxies. Its constituents remain a mystery despite an assiduous search for them over the past three decades. Recent results from the satellite-based PAMELA experiment show an excess in the positron fraction at energies between 10 and $100 \mathrm{GeV}$ in the secondary cosmic ray spectrum. Other experiments, namely ATIC, HESS and FERMI, show an excess in the total electron $\left(e^{+}+e^{-}\right)$ spectrum for energies greater than $100 \mathrm{GeV}$. These excesses in the positron fraction as well as the electron spectrum can arise in local astrophysical processes like pulsars, or can be attributed to the annihilation of the dark matter particles. The latter possibility gives clues to the possible candidates for the dark matter in galaxies and other astrophysical systems. In this article, we give a report of these exciting developments.
\end{abstract}

Keywords. Dark matter; cosmic rays; beyond Standard Model.

PACS Nos 95.35.+d; 96.50.Vg; 12.60.-i

\section{Introduction}

The evidence for the existence of dark matter in various astrophysical systems has been gathering over the past three decades. It is now widely-recognized that the presence of dark matter is required to explain the observations of galaxies and other astrophysical systems on larger scales. The clearest support for the existence of dark matter comes from the now well-known observation of nearly flat rotation curves or constant rotation velocity in the outer parts of galaxies [1,2]. Surprisingly, the rotation velocity is observed to remain almost constant till the last point at which it can be measured [2a]. The simple principle of rotational equilibrium tells that the amount of dark mass to visible mass must increase at larger radii. Thus the existence of the dark matter is deduced from its dynamical effect on the visible matter, namely the stars and the interstellar gas in galaxies. 


\section{Debtosh Chowdhury, Sudhir K Vempati and Chanda J Jog}

The presence of dark matter in the elliptical galaxies is more problematic to ascertain since these do not contain much interstellar hydrogen gas which can be used as a tracer of their dynamics, and also because these galaxies are not rotationally supported. Instead, these galaxies are supported by pressure or random motion of stars (see Binney [3] for details of physical properties of the spiral and elliptical galaxies). As a result, the total mass cannot be deduced using the rotation curve for elliptical galaxies. Instead, the motions of planetary nebulae which arise from old, evolved stars, as well as lensing, have been used to trace the dark matter [4]. The fraction of dark matter at four effective radii is still uncertain with values ranging from $20 \%$ to $60 \%$ given in the literature, for the extensively studied elliptical galaxy NGC 3379 [5].

Historically, the first evidence for the unseen or dark matter was found in clusters of galaxies. Assuming the cluster to be in a virial equilibrium, the total or the virial mass can be deduced from the observed kinematics. Zwicky [6] noted that there is a discrepancy of a factor $\sim 10$ between the observed mass in clusters of galaxies and the virial mass deduced from the kinematics. In other words, the random motions are too large for the cluster to be bound so that a substantial amount of dark matter $(\sim 10$ times the visible matter in galaxies) is needed for the clusters of galaxies to remain bound. This discrepancy remained a puzzle for over four decades, and was only realized as a part of the general trend after the galactic-scale dark matter was discovered in the late 1970s.

On the much larger cosmological scale, there has been some evidence for non-baryonic dark matter from theoretical estimates of primordial elements during Big Bang Nucleosynthesis and measurements of them, particularly, primordial deuterium. Accurate measurements of the cosmic microwave background radiation (CMBR) could also give information about the total dark matter relic density of the Universe. The satellite-based COBE experiment was one of the first experiments to provide accurate 'mapping' of the CMBR [7]. The recent high precision determination of the cosmological parameters using Type I supernova data [8] as well as precise measurements of the cosmic background radiation by the WMAP Collaboration $[9,10]$ has pinpointed the total relic dark matter density in the early universe with an accuracy of a few percent. Accordingly, dark matter forms about $26 \%$ of all the matter density of the Universe, with visible matter about $4 \%$ and the dark energy roughly about $70 \%$. This is known as $\Lambda$ CDM model where $\Lambda$ is dark energy denoted by the Einstein's constant and CDM is Cold Dark Matter [11].

Numerical simulations for the currently popular scenario of galaxy formation, based on the $\Lambda \mathrm{CDM}$ model, predicts a universal profile for the dark matter in halos of spherical galaxies [12]. While this model was initially successful, over the years many discrepancies between the predictions from it and the observations have been pointed out. The strongest one is the 'cusp-core' issue of the central mass distribution. Navarro et al [12] predicted a cuspy [12a] central mass distribution, but the observations of rotation curves of central regions of galaxies, especially the low surface brightness galaxies, when modelled showed a flat or cored density distribution [13].

Another significant alternative to the dark matter which can be used to explain the rotation curves of the galaxies and clusters was proposed earlier by Milgrom. He claimed that for low accelerations [14], Newtonian law has to be modified by the addition of a small repulsive term. This is known as MOND (MOdified Newtonian Dynamics). Initially this 
idea was not taken seriously by the majority of astrophysics community, but it gained more acceptance in recent years. For example, some of the standard features seen in galaxies such as the frequency of bars can be better explained under the MOND paradigm (see Tiret et al [15]). For a summary of the predictions and comparisons of these two alternatives (dark matter and MOND), see Combes et al [16].

So far the most direct empirical evidence for the existence of dark matter, and hence the evidence against MOND, comes from the study of the so-called Bullet cluster [17]. This is a pair of galaxies undergoing a supersonic collision at a redshift of $\sim 0.3$. The main visible baryonic component in clusters is hot X-ray emitting gas. In a supersonic collision, this hot gas would collide and remain at the centre of mass of the colliding system while the stars will just pass through since they occupy a small volume [17a].

In the Bullet cluster, the gravitational potential as traced by the weak-lensing shows peaks that are separated from the central region traced by the hot gas. In MOND, these two would be expected to coincide [17b], since the gravitational potential would trace the dominant visible component namely the hot gas, while if there is dark matter it would be expected to peak at the location of the stellar component in the galaxies. The latter case is what has been observed as shown in figure 1 of [17]. For the rest of the article, the MOND explanation will not be considered, instead we shall take the viewpoint that the flat rotation curves of galaxies and clusters at large radii are an evidence for the existence of dark matter. Furthermore, we believe that the dark matter explanation is much simpler and more natural compared to the MOND explanation. The rest of the discussion will be pertinent to dark matter.

Dark Matter on the other hand, despite being postulated over three decades ago; there is no consensus yet about its constituents. This has been summarized well in many review articles. Examples that span from the earlier to the recent times on this topic are given in refs $[21,22]$. Over the years, both astrophysicists as well as particle physicists have speculated on the nature of dark matter.

The baryonic dark matter in the form of low-mass stars, binary stars, or Jupiter-like massive planets were ruled out before (see [21] for a summary). From the amount of dark matter required to explain the flat rotation curves, it can be shown that the number densities required of these possible constituents would be large, and hence it would be hard to hide these massive objects. Because, if present in these forms, they should have been detected either from their absorption or from their emission signals. It has also been proposed that the galactic dark matter could be in the form of dense, cold molecular clumps [23], though this has not yet been detected. This alternative cannot be expected to explain the dark matter necessary to 'fit' the observations of clusters, or indeed the elliptical galaxies since the latter have very little interstellar gas.

There is also a more interesting possibility of the dark matter being essentially of baryonic nature, but due to the dynamics of the QCD phase transition in the early Universe which left behind a form of cold quark-gluon plasma, the baryon number content of the dark matter is hidden from us. This idea was first proposed by Witten in 1984 [24], who called these quantities as quark nuggets. An upper limit on the total number of baryons in a quark nugget is determined by the baryon-to-photon ratio in the early Universe (see for example [25]). Taking these constraints into consideration, it is possible to fit the 


\section{Debtosh Chowdhury, Sudhir K Vempati and Chanda J Jog}

observed relic density with a mass (density) distribution of the quark nuggets [26] (for the observational possibilities of such quark nuggets, see [27]).

From a more fundamental point of view, it is not clear what kind of elementary particle could form dark matter. The Standard Model of particle physics describes all matter to be made up of quarks and leptons of which neutrinos are the only ones which can play the role of dark matter as they are electrically neutral. However, with the recent indications from various neutrino-oscillation experiments putting the Standard Model neutrino masses in the range $\lesssim 1 \mathrm{eV}$ [28] they will not form significant amount of dark matter. There could, however, be non-standard sterile neutrinos with masses of the order of keV$\mathrm{MeV}$ which could form warm [28a] dark matter (for reviews, see [30,31). Cold dark matter (CDM), on the other hand, is favoured over the warm dark matter by the hierarchical clustering observed in numerical simulations for large scale structure formation [29]. Recent analysis including X-ray flux observations from Coma Cluster and Andromeda Galaxy have shown that the room for sterile neutrino warm dark matter is highly constrained [32]. However, if one does not insist that the total relic dark matter density is due to sterile neutrinos, then it is still possible that they form a sub-dominant warm component of the total dark matter [33] relic density [33a].

The Standard Model (SM) thus, needs to be extended to incorporate a dark matter candidate. The simplest extensions would be just to include a new particle which is a singlet under the SM gauge group (i.e., does not carry the Standard Model interactions). Further, we might have to impose an additional symmetry under which the dark matter particle transforms non-trivially to keep it stable or at least sufficiently long-lived with a lifetime typically larger than the age of the Universe. Some of the simplest models would just involve adding additional light $(\sim \mathrm{GeV})$ scalar particles to the SM and with an additional $U(1)$ symmetry [37]. Similar extensions of SM can be constructed with fermions too $[38,39]$. An interesting aspect of these set of models is that they can be tested at the existing $e^{+} e^{-}$colliders, like for example, the one at present at Frascati, Italy [40]. A heavier set of dark matter candidates can be achieved by extending the Higgs sector by adding additional Higgs scalar doublets, which go by the name of inert Higgs models $[41,42]$. In this extension, there is an additional neutral Higgs boson which does not have SM gauge interactions (hence inert), which can be a dark matter candidate. With the inclusion of this extra inert Higgs doublet, the SM particle spectrum has some added features: it can evade the 'naturalness problem' up to $1.5 \mathrm{TeV}$ while preserving the perturbativity of Higgs couplings up to high scales, and further it is consistent with the electroweak precision tests [43].

On the other hand, there exist extensions of the Standard Model (generally labelled beyond Standard Model (BSM) physics) which have been constructed to address a completely different problem called the hierarchy problem. The hierarchy problem addresses the lack of symmetry for the mass of the Higgs boson in SM and the consequences of this in the light of the large difference of energy scales between the weak interaction scale $\left(\sim 10^{2} \mathrm{GeV}\right)$ and the quantum gravity or grand unification scale $\left(\sim 10^{16} \mathrm{GeV}\right)$. Such a huge difference in the energy scales could destabilize the Higgs mass due to quantum corrections. To protect the Higgs mass from these dangerous radiative corrections, new theories such as supersymmetry, large extra dimensions and little Higgs have been proposed. It turns out that most of the BSM physics models contain a particle which can be 


\section{Results from PAMELA, ATIC and FERMI: Pulsars or dark matter?}

the dark matter. A few examples of these theories and the corresponding candidates for dark matter are as follows:

(i) Axions are pseudoscalar particles which appear in theories with Peccei-Quinn symmetry $[44,45]$ proposed as solution to the strong CP problem of the Standard Model. They also appear in superstring theories which are theories of quantum gravity. The present limits on axions are [46] extremely strong from astrophysical data. In spite of this, there is still room for axions to form a significant part of the dark matter relic density.

(ii) Supersymmetric theories $[47,48]$ which incorporate fermion-boson interchange symmetry are proposed as extensions of SM to protect the Higgs mass from large radiative corrections. The dark matter candidate is the lightest supersymmetric particle (LSP) which is stable or sufficiently long-lived as mentioned before [48a]. Depending on how supersymmetry is broken [49], there are several possible dark matter candidates in these models. In some models, the lightest supersymmetric particle and hence the dark matter candidate is a neutralino. The neutralino is a linear combination of superpartners of $Z, \gamma$ as well as the neutral Higgs bosons [49a]. The other possible candidates are the superpartner of the graviton, called the gravitino and the superpartners of the axinos, the scalar saxion and the fermionic axino. These particles also can explain the observed relic density [52].

(iii) Other classic extensions of the Standard Model, either based on additional space dimensions or larger symmetries, also have dark matter candidates. In both versions of the extra dimensional models, i.e., the Arkani-Hamed, Dimopoulos, Dvali (ADD) $[53,54]$ and Randall-Sundrum (RS) [55,56] models, the lightest Kaluza-Klein particle [54a] can be considered as the dark matter candidate [57-60]. Similarly, in the little Higgs models where the Higgs boson is a pseudo-Goldstone boson of a much larger symmetry; a symmetry called T-parity [61] assures us a stable and neutral particle which can form the dark matter. Very heavy neutrinos with masses of $\mathcal{O}(100 \mathrm{GeV}-1 \mathrm{TeV})$ can also naturally appear within some classes of Randall-Sundrum and little Higgs models. Under suitable conditions, these neutrinos can act like cold dark matter (for a recent study, see [62]). In addition to these particles, more exotic candidates like simpzillas [63] and wimpzillas [64] with masses close to the GUT scale $\left(\sim 10^{15} \mathrm{GeV}\right)$ have also been proposed in the literature. Indirect searches like ICECUBE [65] (discussed below) already have strong constraints on simpzillas.

\section{Dark matter experiments}

If the dark matter candidate is indeed a new particle and it has interactions other than gravitational interactions [65a], then the most probable interactions it could have are the weak interactions [65b]. This weakly interacting particle, dubbed as weakly interacting massive particle (WIMP) could interact with ordinary matter and leave traces of its nature. There are two ways in which the WIMP can be detected:

(i) Direct detection. Here one looks for the interaction of the WIMP on a target, the target being typically nuclei in a scintillator. It is expected that the WIMPs present all over the Galaxy scatter off the target nuclei once in a while. Measuring the recoil of the nuclei in these rarely occurring events would give us information about the properties of 
the WIMP. The scattering cross-section would depend on whether it was elastic or inelastic and is a function of the spin of the WIMP [65c]. More than 20 experiments are located all over the world, which are currently looking for WIMP through this technique. Some of them are DAMA, CDMS, CRESST, CUORICINO, DRIFT etc.

(ii) Indirect detection. When WIMPs cluster together in the galatic halo, they can annihilate with themselves giving rise to electron-positron pairs, $\gamma$-rays, proton-anti-protonpairs, neutrinos etc. The flux of such radiation is directly proportional to the annihilation rate and the WIMP matter density. Observation of this radiation could lead to information about the mass and the cross-section strength of the WIMPs. At present, there are several experiments which are looking for this radiation [66a]: (i) MAGIC, HESS, CANGAROO, FERMI/GLAST, EGRET etc. look for the $\gamma$-ray photons, (ii) HEAT, CAPRICE, BESS, PAMELA, AMS can observe anti-protons and positron flux and (iii) highly energetic neutrinos/cosmic rays $-\mathrm{a}$ few $\mathrm{TeV}$ to multi-TeV can be observed by large detectors like AMANDA, ANTARES, ICECUBE etc. (for a more detailed discussion, see $[46,67]$ ).

Over the years, there have been indications of the presence of the dark matter through both direct and indirect experiments. The most popular among these signals are INTEGRAL and DAMA results (for a nice discussion on these topics, please see [68]). International Gamma-Ray Astrophysics Laboratory (INTEGRAL) is a satellite-based experiment looking for $\gamma$-rays in outer space. A very bright emission of the $511 \mathrm{keV}$ photons from the galactic bulge [69] at the centre was observed in 2003. The $511 \mathrm{keV}$ line is special as it is dominated by $e^{+} e^{-}$annihilations via the positronium. The observed rate of $(3-15) \times 10^{42}$ positrons $/ \mathrm{s}$ in the inner galaxy was much larger than the expected rate from pair creation via cosmic ray interactions with the interstellar medium in the galactic bulge by orders of magnitude [69a]. Further, the signal is almost spherically symmetric with very little positrons from galactic bulge contributing to it [70]. Several explanations have been put forward to explain this excess. Astrophysical entities like hypernovae, $\gamma$-ray bursts and X-ray binaries have been proposed as the likely objects contributing to this excess. On the other hand, this signal can also be attributed to the presence of dark matter which can annihilate itself giving rise to electron-positron pairs. To explain the INTEGRAL signal in terms of dark matter, extensions of Standard Model involving light $(\sim \mathrm{MeV}-\mathrm{GeV})$ particles and light gauge bosons $(\sim \mathrm{GeV})$ are ideally suited. These models which have been already reviewed in the previous section, can be probed directly at the existing and future $e^{+} e^{-}$colliders and hence can be tested. Until further confirmation from either future astrophysical experiments or through ground-based colliders comes about, the INTEGRAL remains an 'anomaly' as of now.

While INTEGRAL is an indirect detection experiment, DAMA (DArk MAtter) is a direct detection experiment located in the Gran Sasso mountains of Italy. The target material consists of highly radio pure $\mathrm{NaI}$ crystal scintillators; the scintillating light from WIMP-nucleon scattering and recoil is measured. The experiment looks for an annual modulation of the signal as the Earth revolves around the Sun [71]. Such modulation of the signal is due to the gravitational effects of the Sun as well as rotatory motion of the Earth [71a]. DAMA and its upgraded version DAMA/LIBRA have collected data for seven annual cycles and four annual cycles, respectively [71b]. Together they have reported an annual modulation at $8.2 \sigma$ confidence level. If confirmed, the DAMA results would be the first direct experimental evidence for the existence of WIMP dark matter 
particle. However, the DAMA results became controversial as this positive signal has not been confirmed by other experiments like XENON and CDMS, which have all reported null results in the spin-independent WIMP-nucleon scattering signal region.

The Xenon 10 detector at Gran Sasso Laboratories uses a xenon target for measuring simultaneously the scintillation and ionization produced by the scattering of the dark matter particle. The simultaneous measurement reduces the background significantly down to $4.5 \mathrm{keV}$. With a fiducial mass of $5.4 \mathrm{~kg}$, they set an upper limit of WIMP-nucleon spinindependent cross-section to be $8.8 \times 10^{-44} \mathrm{~cm}^{2}$ for a WIMP mass of $100 \mathrm{GeV}$ [73]. An upgraded version Xenon 100 having about double the fiducial mass has started taking data from October 2009. In the first results, they present null results, with upper limits of about $3.4 \times 10^{-44} \mathrm{~cm}^{2}$ for $55 \mathrm{GeV}$ WIMPs [74]. These results severely constrain interpretation of the DAMA results in terms of an elastic spin-independent WIMP-nucleon scattering.

The Cryogenic Dark Matter Search (CDMS) experiment has 19 germanium detectors located at the underground Soudan Mine, USA. It is maintained at temperature $\sim 40 \mathrm{mK}$ (milli-Kelvin). Nuclear recoils can be 'seen' by measuring the ionization energy in the detector. Efficient separation between electron recoils and nuclear recoils is possible by employing various techniques like signal timing and measuring the ratios of the ionization energies. Similar to xenon, this experiment [75] too reported null results in the signal region [72a] and put an upper limit $\sim 4.6 \times 10^{-44} \mathrm{~cm}^{2}$ on the WIMP-nucleon cross-section for a WIMP mass of around $60 \mathrm{GeV}$.

The Cryogenic Germanium Neutrino Technology (CoGeNT) Collaboration runs another recent experiment which uses ultra-low noise germanium detectors. It is located in the Soudan Mine, USA. The experiment has one of the lowest backgrounds below 3 $\mathrm{keVee}$ (keV electron equivalent (ee) ionization energy). It can further go down to 0.4 keVee, the electron noise threshold. Initial runs have again reported null results [76] consistent with the observed background. At this point, the experiment did not have the sensitivity to confirm/rule out the DAMA results. Later runs, however, have shown some excess events over the expected background in the low-energy regions [77]. Even though the collaboration could not find a suitable explanation for this excess (as of now), there is a possibility of these excess events having their origins in a very light WIMP dark matter particle. But, care should be taken before proceeding with this interpretation as the CoGeNT Collaboration does not distinguish between electron recoils and nucleon recoils [78].

In the light of these experimental results, the DAMA results are hard to explain. An alternative to make the DAMA results consistent with other experiments is to include an effect called 'channelling' which will be present only in the NaI crystals which DAMA uses. But, even the inclusion of this effect does not improve the situation significantly. To summarize, the situation for various interpretations of the WIMP-nucleon cross-section is shown as follows. For elastic spin-independent (eSI) interpretation, the DAMA regions are excluded by both CDMS as well as Xenon 10 irrespective of whether one considers the channelling effect or not. It is also hard to reconcile DAMA results with CoGeNT in this case. For elastic spin-dependent (eSD) interpretation, the DAMA and CoGeNT results (though consistent with each other) are in conflict with other experiments. For an interpretation in terms of WIMP-proton scattering, the results are in conflict with several experiments like SIMPLE, PICASSO etc. On the other hand, an 


\section{Debtosh Chowdhury, Sudhir K Vempati and Chanda J Jog}

interpretation in terms of WIMP-neutron scattering is ruled out by XENON and CDMS data. For the inelastic dark matter interpretations, spin-independent cross-section with a medium mass $(\sim 50 \mathrm{GeV})$ WIMP is disfavoured by CRESST as well as CDMS data. For a low-mass (close to $10 \mathrm{GeV}$ ) WIMP, with the help of channelling in the NaI crystals, it is possible to explain the DAMA results, in terms of spin-independent inelastic dark matternucleon scattering. But, the relevant parameters (dark matter mass and mass splittings) should be fine tuned, and further the WIMP velocity distribution in the Galaxy should be close to the escape velocity. Inelastic spin-dependent interpretation of the DAMA results is a possibility (because it can change relative signals at different experiments [79]) which does not have significant constraints from other experiments. But, it has been shown [78] that inelastic dark matter, either with spin-dependent or spin-independent interpretation of the DAMA results, is difficult to reconcile with the CoGeNT results, unless one introduces substantial exponential background in the CoGeNT data.

\section{The data}

The focus of the present topical review is a set of new experimental results which have appeared over the past one year. In terms of the discussion in the previous section, these experiments follow 'indirect' methods to detect dark matter. The data from these experiments seem to be pointing to either 'discovery' of the dark matter or some hitherto non-understood new astrophysics being operative within the vicinity of our Galaxy. The four main experiments which have led to this excitement are; (i) PAMELA [80], (ii) ATIC [81], (iii) HESS [82] and (iv) FERMI [83]. All these experiments involve international collaborations spanning several nations. While PAMELA and FERMI are satellite-based experiments, ATIC is a balloon-borne experiment and HESS is a ground-based telescope. These experiments are technologically more improved compared to the previous generation experiments of similar type. The HESS experiment has a factor $~ 10$ improvement in $\gamma$-ray flux sensitivity over previous experiments largely because of its superior rejection of the hadronic background. Similarly, ATIC is the next-generation balloon-based experiment equipped to have higher resolution as well as larger statistics. Similar features also hold for the satellite-based experiments, PAMELA and FERMI. It should be noted that the satellite-based experiments have some inherent advantages over the balloon-based ones. First, they have enhanced data taking period, unlike the balloon-based ones which can take data only for short periods. Furthermore, these experiments do not have problems with the residual atmosphere on the top of the instrument which plagues the balloon-based experiments.

The satellite-based Payload for Anti-Matter Matter Exploration and Light-nuclei Astrophysics (PAMELA) collects cosmic ray protons, anti-protons, electrons, positrons and light nuclei like helium and anti-helium. One of the main strengths of PAMELA is that it could distinguish between electrons and anti-electrons, protons and anti-protons and measure their energies accurately. The sensitivity of the experiment in the positron channel is $\sim 300 \mathrm{GeV}$ and in the anti-proton channel $\sim 200 \mathrm{GeV}$. Since it was launched in June 2006, it was placed in an elliptical orbit at an altitude ranging between 350 and $610 \mathrm{~km}$ with an inclination of $70^{\circ}$. Data for about 500 days were analysed and presented recently. The present data from $1.5 \mathrm{GeV}$ to $100 \mathrm{GeV}$ have been published in Nature 
[80]. In this paper, PAMELA reported an excess of positron flux compared to earlier experiments. In the left panel of figure 1, we see PAMELA results along with other existing ones. The $y$-axis is given by $\phi\left(e^{+}\right) /\left(\phi\left(e^{-}\right)+\phi\left(e^{+}\right)\right)$, where $\phi$ represents the flux of the corresponding particle. The results of PAMELA are consistent with the earlier experiments up to $20 \mathrm{GeV}$, taking into consideration the solar modulations between the times of PAMELA and previous experiments. Particles with energies up to $20 \mathrm{GeV}$ are strongly effected by solar wind activity which varies with solar cycle. On the other hand, PAMELA has data from $10 \mathrm{GeV}$ to $100 \mathrm{GeV}$, which show an increase in the positron flux (figure 1). The only other experimental data in this energy regime (up to $40 \mathrm{GeV}$ ) are the AMS and HEAT, which even though having large errors, are consistent with the excess seen by PAMELA. In the low-energy regime, many of the other experiments are in accordance with each other but have large error bars.

Cosmic ray positrons at these energies are expected to be from secondary sources, i.e. as a result of the interactions of the primary cosmic rays (mainly protons and electrons) with interstellar medium. The flux of this secondary sources can be estimated by numerical simulations. Several numerical codes are available to compute the secondary flux, the most popular publicly available being GALPROP [91,92] and CRPropa [93]. These codes compute the effects of interactions and energy losses during cosmic ray propagation

a

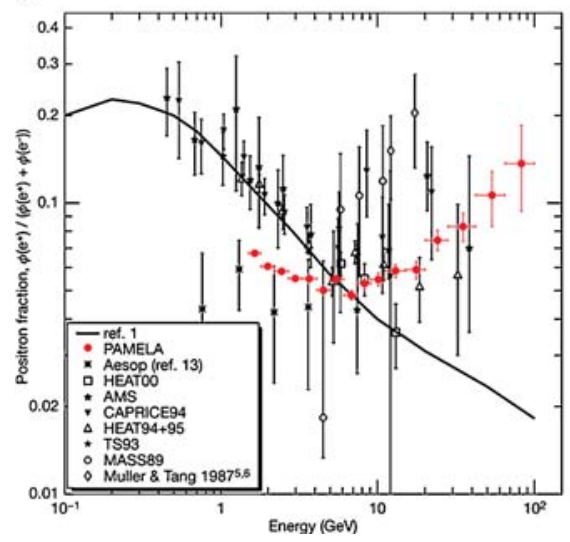

b

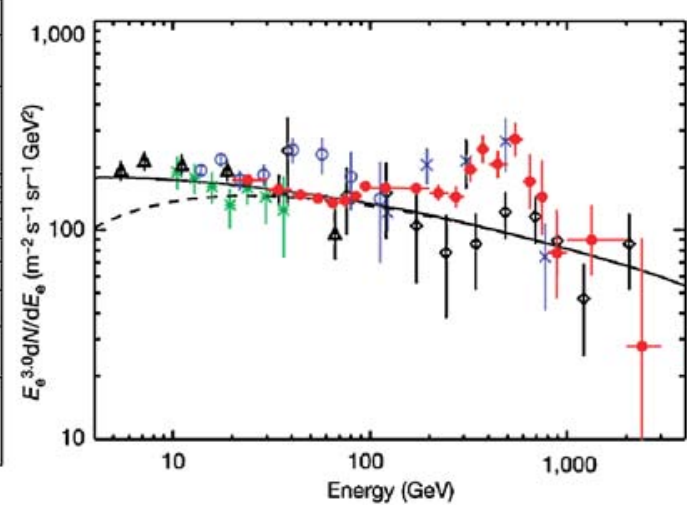

Figure 1. Results from PAMELA and ATIC with theoretical models. The left panel shows PAMELA [80] positron fraction along with theoretical model. The solid black line shows a calculation by Moskalenko and Strong [84] for pure secondary production of positrons during the propagation of cosmic rays in the Galaxy. The right panel shows the differential electron energy spectrum measured by ATIC [81] (red circles) compared with other experiments and also with theoretical prediction using the GALPROP [85] code (solid line). The other data points are from AMS [86] (green stars), HEAT [87] (open triangles), BETS [88] (open circles), PPB-BETS [89] (blue crosses) and emulsion chambers (open diamonds) and the dashed curve at the beginning is the spectrum of solar modulated electron. All the data points have uncertainties of one standard deviation. The ATIC spectrum is scaled by $E_{e}^{3.0}$. (The figures of PAMELA and ATIC are reproduced from their original papers cited above.) 


\section{Debtosh Chowdhury, Sudhir K Vempati and Chanda J Jog}

within galactic medium taking into account the galactic magnetic fields. GALPROP solves the differential equations of motion using either a 2D grid or a 3D grid whereas CRPropa does the same using a 1D or a 3D grid. While GALPROP contains a detailed exponential model of the galactic magnetic fields, CRPropa implements only extragalactic turbulent magnetic fields. In particular, CRPropa is not optimized for convoluted galactic magnetic fields. For this reason, GALPROP is best-suited for solving diffusion equations involving low-energy $(\mathrm{GeV}-\mathrm{TeV})$ cosmic rays in galactic magnetic fields.

The main input parameters of the GALPROP code are the primary cosmic ray injection spectra, the spatial distribution of cosmic ray sources, the size of the propagation region, the spatial and momentum diffusion coefficients and their dependencies on particle rigidity. These inputs are mostly fixed by observations: the interstellar gas distribution is based on observations of neutral atomic and molecular gas, ionized gas; cross-sections and energy fitting functions are build from nuclear data sheets (based on Las Almos Nuclear compilation of nuclear cross-sections and modern nuclear codes) and other phenomenological estimates. Interstellar radiation fields and galactic magnetic fields are based on various models given in literature. The uncertainties in these inputs would constitute the main uncertainties in the flux computation from GALPROP [93a]. Recently, a new code called CRT which emphasizes the minimization of the computation time was introduced. Here most of the input parameters are user-defined [95]. Finally, using the popular Monte Carlo routine GEANT [96] one can construct cosmic ray propagation code as has been done by [97,98]. On the other hand, dark matter relic density calculators like DARKSUSY [99] compute cosmic ray propagation in the galaxies required for indirect searches of dark matter. It is further interfaced with GALPROP.

In summary, GALPROP is the most suitable for understanding PAMELA and ATIC data which are mostly in the $\mathrm{GeV}-\mathrm{TeV}$ range. The results from these experiments do not vary much if one chooses to use a GEANT simulation. In fact, most of the experimental collaborations use GALPROP for their predictions of secondary cosmic ray spectrum. In the left panel of figure 1, the expectations based on GALPROP are given as a solid line running across the figure. From the figure it is obvious that PAMELA results show that the positron fraction increases with energy compared to what GALPROP expects. The excess in the positron fraction measured by PAMELA with respect to GALPROP indicates that this result is due to new primary rather than secondary sources [99a]. This new primary source could be either dark matter decay/annihilation or a nearby astrophysical object like a pulsar. Before going to the details of the interpretations, let us summarize the results from ATIC and FERMI.

Advanced Thin Ionization Calorimeter (ATIC) is a balloon-borne experiment to measure energy spectrum of individual cosmic ray elements within the region of $\mathrm{GeV}$ up to almost a $\mathrm{TeV}$ (thousand $\mathrm{GeV}$ ) with high precision. As mentioned, this experiment was designed to be a high-resolution and high-statistics experiment in this energy regime compared to the earlier ones. ATIC measures all the components of the cosmic rays such as electrons, protons (and their anti-particles) with high energy resolution, distinguishing electrons and protons. ATIC (right panel in figure 1) presented its primary cosmic ray electron $\left(e^{-}+e^{+}\right)$spectrum between the energies $3 \mathrm{GeV}$ and $2.5 \mathrm{TeV}$ [100a]. The results show that the spectrum while agreeing with the GALPROP expectations up to $100 \mathrm{GeV}$, show a sharp increase above $100 \mathrm{GeV}$. The total flux increases up to about $600 \mathrm{GeV}$ where 
it peaks and then sharply falls to about $800 \mathrm{GeV}$. Thus, ATIC sees an excess of the primary cosmic ray $\left(e^{-}+e^{+}\right)$spectrum in the energy range $300-800 \mathrm{GeV}$. The rest of the spectrum is consistent with the expectations within the errors. What is interesting about such peaks in the spectrum is that, if they are confirmed they can point towards a Breit-Wigner resonance in dark matter annihilation cross-section with a lifetime as given by its width. This possibility is severely constrained by the data from the FERMI experiment, which is discussed in $\S 4$.

Another ground-based experiment sensitive to cosmic rays within this energy range is HESS which can measure $\gamma$-rays from few hundred $\mathrm{GeV}$ to few $\mathrm{TeV}$. This large reflecting array telescope operating from Namibia has presented data (figure 2) from $600 \mathrm{GeV}$ to about $5 \mathrm{TeV}$. It could confirm neither the 'peaking' like behaviour at $600 \mathrm{GeV}$ nor the sharp cut-off at $800 \mathrm{GeV}$ of the ATIC data. The ATIC results can be made consistent with those of HESS. This requires a $15 \%$ overall normalization of the HESS data. Such a normalization is well within the uncertainty of the energy resolution of HESS. Note that HESS data do not have a sharp fall around $800 \mathrm{GeV}$.

The Large Area Telescope (LAT) is one of the main components of the Fermi gamma ray space telescope, which was launched in June 2008. Because of its high resolution and high statistical capabilities, it is one of the most anticipated experiments in recent times. Fermi can measure $\gamma$-rays between $20 \mathrm{MeV}$ and $300 \mathrm{GeV}$ with high accuracy and primary cosmic ray electron $\left(e^{-}+e^{+}\right)$flux between $20 \mathrm{GeV}$ and $1 \mathrm{TeV}$. The energy resolution averaged over the LAT acceptance is $11 \%$ full-width-at-half-maximum

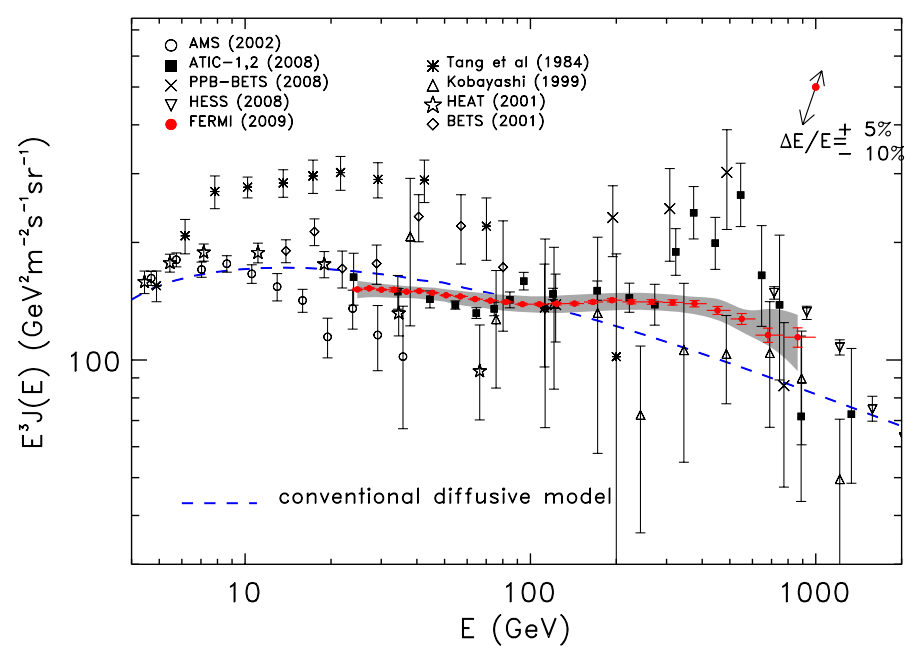

Figure 2. The Fermi LAT CR electron spectrum. The red circles show the data from Fermi along with the gray bands showing systematic errors. The dashed line corresponds to a theoretical model by Moskalenko et al [90]. (The figure of FERMI is reproduced from their original paper cited above.)

Pramana - J. Phys., Vol. 76, No. 1, January 2011 


\section{Debtosh Chowdhury, Sudhir K Vempati and Chanda J Jog}

(FWHM) for 20-100 GeV, increasing to 13\% FWHM for 150-200 GeV. The photon angular resolution is less than $0.1^{\circ}$ over the energy range of interest (68\% containment). The FERMI-LAT Collaboration has recently published its six-month data on the primary cosmic ray electron flux. More than 4 million electron events above $20 \mathrm{GeV}$ were selected in survey (sky scanning) mode from 4 August 2008 to 31 January 2009. The systematic error on the absolute energy of the LAT was determined to be between $-10 \%$ and $+5 \%$ for 20-300 GeV. (For more details on the errors, see Table I in [83]). In figure 2 we reproduce the result obtained by the FERMI Collaboration. They found the primary cosmic ray electron spectrum more or less along the expected lines up to $100 \mathrm{GeV}$ (it is slightly below the expected flux between 10 and $50 \mathrm{GeV}$, but above $100 \mathrm{GeV}$ ), there is a strong signal for an excess of the flux ranging up to $1 \mathrm{TeV}$. The FERMI data thus confirmed the excess in the electron spectrum which was seen by ATIC. The excess however had a much flatter profile with respect to the peak seen by ATIC. Thus, ATIC could in principle signify a 'resonance' in the spectrum, whereas FERMI could not. However, in comparing both the spectra from the figures presented above, one should keep in mind that the FERMI excess is in the total electron spectrum $\left(e^{+}+e^{-}\right)$, whereas the ATIC data is presented in terms of positron excess only. If the excess in FERMI is caused by the excess only through excess positrons, one should expect that the FERMI spectra can have similar 'peak'-like behaviour at $600 \mathrm{GeV}$. From figure 2, where both FERMI and ATIC data are presented, we see that the ATIC data points are far above that of FERMI's.

\section{The interpretations}

Lets now summarize the experimental observations [98] which would require an interpretation:

- The excess in the flux of positron fraction $\left(\phi\left(e^{+}\right) / \phi\left(e^{-}\right)+\phi\left(e^{+}\right)\right)$measured by PAMELA up to $100 \mathrm{GeV}$.

- The lack of excess in the anti-proton fraction measured by PAMELA up to $100 \mathrm{GeV}$.

- The excess in the total flux $\left(\phi\left(e^{-}\right)+\phi\left(e^{+}\right)\right)$in the spectrum above $100 \mathrm{GeV}$ shown by FERMI, HESS etc. But below $100 \mathrm{GeV}$, the measurements have been consistent with GALPROP expectations.

- The absence of 'peaking'-like behaviour as seen by ATIC, which indicates a longlived particle, in the total electron spectrum measured by FERMI.

Two main interpretations have been put forward: (a) a nearby astrophysical source which has a mechanism to accelerate particles to high energies and (b) a dark matter particle which decays or annihilates leading to excess of electron and positron flux. Which of the interpretations is valid will be known in the coming years with enhanced data from both PAMELA and FERMI. Let us now turn to both the interpretations.

Pulsars and supernova shocks have been proposed as likely astrophysical local sources of energetic particles that could explain the observed excess of the positron fraction $[101,102]$. In the high magnetic fields present in the pulsar magnetosphere, electrons can be accelerated which can induce an electromagnetic cascade through the emission of curvature radiation [102a]. This can lead to a production of high-energy photons above the 


\section{Results from PAMELA, ATIC and FERMI: Pulsars or dark matter?}

threshold for pair production; and on combining with the number density of pulsars in the Galaxy, the resulting emission can explain the observed positron excess [101]. The energy of the positrons tells us about the site of their origin and propagation history [104]. The cosmic ray positrons above $1 \mathrm{TeV}$ can be primary and arise due to a source like an young plusar within a distance of $100 \mathrm{pc}$ [105]. This can also naturally explain the observed anisotropy, as argued for two of the nearest pulsars, namely B0656 +14 and the Geminga [106,107]. On a similar note, diffusive shocks as in a supernova remnant hardens the spectrum, and this process can explain the observed positron excess above 10 $\mathrm{GeV}$ as seen from PAMELA [108].

Another possible astrophysical source that has been proposed is the pion production during acceleration of hadronic cosmic rays in the local sources [102]. It was argued [109] that the measurement of secondary nuclei produced by cosmic ray spallation can confirm whether this process or pulsars are more important as the production mechanism. It is shown that the present data from ATIC-II support the hadronic model and can account for the entire positron excess observed.

If the excess observed by PAMELA, HESS and FERMI is not due to some yet not fully-understood astrophysics but is a signature of the dark matter, then there are two main processes through which such an excess can occur:

(i) The annihilation of dark matter particles into Standard Model (SM) particles.

(ii) The decay of the dark matter particle into SM particles.

Interpretation in terms of annihilating dark matter, however, leads to conflicts with cosmology. The observed excesses in the PAMELA/FERMI data would set a limit on the product of annihilation cross-section and the velocity of the dark matter particle in the Galaxy (for a known dark matter density profile). Annihilation of the dark matter particles also happens in the early Universe with the same cross-section but at much larger velocities for particles (about 1000 times the particle velocities in Galaxies). The resultant relic density is not compatible with observations. The factor $\sim 1000$ difference in the velocities should somehow be compensated in the cross-sections. This can be compensated by considering 'boost' factors for the particles in the Galaxy which can enhance the cross-section by several orders of magnitude. The boost factors essentially emanate by assuming local substructures for the dark matter particles, like clumps of dark matter and are typically free parameters of the model [110]. Another mechanism which is called Sommerfeld mechanism can also enhance the annihilation cross-sections. For very heavy dark matter (with masses much greater than the relevant gauge boson masses) trapped in the galactic potential, non-perturbative effects could push the annihilation cross-sections to much larger values. For $S U(2)$ charged dark matter, the masses of dark matter particles should be $\gg M_{W}$ [111]. The Sommerfeld mechanism is more general and applicable to other (new) interactions also [112,112a]. Another way of avoiding conflict with cosmology would be to consider non-thermal production of dark matter in the early Universe [112c]. Before the release of FERMI data, the annihilating dark matter model with a very heavy dark matter $\sim \mathcal{O}(2-3) \mathrm{TeV}$ was much in favour for explaining the 'resonance peak' of the ATIC and the excess in PAMELA data. Post-FERMI, whose data do not have sharp rise and fall associated with a resonance, the annihilating dark matter interpretation has been rendered incompatible. Considering possible variations in the local astrophysical 


\section{Debtosh Chowdhury, Sudhir K Vempati and Chanda J Jog}

background profile due to the presence of local cosmic ray accelerator, it has been shown that it is still possible to explain the observed excess, along with FERMI data with annihilating dark matter. The typical mass of the dark matter particle could lie even within sub-TeV region [114-117] and as low as 30-40 GeV [118]. More detailed analysis can be found in [119].

Several existing BSM physics models of annihilating dark matter become highly constrained or ruled out if one requires to explain PAMELA/ATIC and FERMI data. The popular supersymmetric DM candidate neutralino with its annihilating partners such as chargino, stop, stau etc. can explain the cosmological relic density, but not the excess observed by PAMELA/ATIC. Novel models involving a new 'dark force', with a gauge boson having about $1 \mathrm{GeV}$ mass [120], which predominantly decays to leptons together with the so-called Sommerfeld enhancement, seem to fit the data well. The above class of models, which are extensions of Standard Model with an additional $U(1)$ gauge group, caught the imagination of the theorists [121-124]. A similar supersymmetric version of this mechanism where the neutralinos in the MSSM can annihilate to a scalar particle, which can then decay the observed excess in the cosmic ray data [125]. Models involving Type II see-saw mechanism [126] have also been considered recently where neutrino mass generation is linked with the positron excess. In addition to the above, it has been shown that extra dimensional models with KK gravitions can also produce the excess [127,127a]. Models with Nambu-Goldstone bosons as dark matter have been studied in [130].

In the case of decaying dark matter, the relic density constraint of the early Universe is not applicable. However, the lifetime of the dark matter particle (typically of a mass of $\mathcal{O}(1) \mathrm{TeV})$ should be much larger than $\left(\sim 10^{9}\right.$ times $)$ the age of the Universe [98]. Such a particle can fit the data well. A crucial difference in this picture with respect to the annihilation picture is that the decay rate is directly proportional to the density of the dark matter $(\rho)$, whereas the annihilation rate is proportional to its square $\left(\rho^{2}\right)$. The most promising candidate in the decaying dark matter seems to be a fermion (scalar) particle decaying into $W^{ \pm} l^{ \pm}$etc. ( $W^{+} W^{-}$etc.) [131-134]. In terms of the BSM physics, supersymmetric models with a heavy gravitino and small $R$-parity violation have been proposed as candidates for decaying dark matter [135]. A heavy neutralino with $R$-parity violation can also play a similar role [136]. A recent more general model-independent analysis has shown that assuming the GALPROP background, gravitino decays cannot simultaneously explain both PAMELA and FERMI excesses. But, the presence of additional astrophysical sources can change the situation [137]. Independent of the gravitino model, it has been pointed out that the decays of the dark matter particle could be new signals for unification where the dark matter candidate decays through dimension-six operators suppressed by two powers of GUT scale [138-140]. Finally, there has also been some discussion about the possibilities of dark matter consisting of two particles, out of which one is the decaying partner. This goes under the name of 'two-component dark matter' and analysis of this scenario can be seen in [141].

We have so far mentioned just a sample of the theoretical ideas proposed in the literature. Several other equally interesting and exciting ideas have been put forward, but not presented here to avoid lengthy descriptions. 
Results from PAMELA, ATIC and FERMI: Pulsars or dark matter?

\section{Outlook and remarks}

An interesting aspect about the present situation is that, future data from PAMELA and FERMI can distinguish whether the astrophysical interpretation (in terms of pulsars) or the particle physics interpretation in terms of dark matter is valid [142]. PAMELA is sensitive up to $300 \mathrm{GeV}$ in its positron fraction and this together with the measurement of the total electron spectrum can strongly affect the dark matter interpretations. FERMI with its improved statistics can, on the other hand, look for anisotropies within its data [143] which can exist if the pulsars are the origin of this excess. Further measurements of the anti-deuteron can possibly give us a hint about why there is no excess in the anti-proton channel [144]. Similarly, neutrino physics experiments can give us valuable information on the possible models [145]. Finally, the Large Hadron Collider can also give strong hints on the nature of dark matter through direct production [146].

As we have been preparing this note, there has been news from an experiment called cryogenic dark matter search experiment (CDMS-II) [147]. As mentioned before, this experiment conducts direct searches for WIMP dark matter by looking at collisions of WIMPs on supercooled nuclear target material. The present and final analyses of this experiment have shown two events in the signal region, with the probability of observing two or more background events in that region close to $23 \%$. Thus, although these results are positive and encouraging, they are not conclusive. However, these results already set a stringent upper bound on the WIMP-nucleus cross-section for a WIMP mass of around $70 \mathrm{GeV}$. The exclusion plots in the parameter space of WIMP cross-section and WIMP mass are presented in [147]. The interpretations of this positive signal are quite different compared to the signal of PAMELA and FERMI. While PAMELA and FERMI as we have seen would require severe modifications for the existing BSM models of dark matter, CDMS results if confirmed would prefer the existing BSM dark matter candidates like neutralino of the supersymmetry. There are ways of making both PAMELA/FERMI and CDMS-II consistent with dark matter interpretations. But, we shall not discuss it further here. Finally, it is possible to make CDMS-II results consistent with DAMA annual modulation results by assuming a spin-dependent inelastic scattering of WIMP on nuclei [79].

In this paper, we have tried to convey exciting developments which have been happening recently within the interface of astrophysics and particle physics, especially on the most intriguing subjects of our time, namely, the dark matter. Though it has been proposed about sixty years ago, so far we have neither found any conclusive evidence of its existence other than through gravitational interactions, nor of its fundamental composition. Experimental searches which have been going on for decades have not bore fruit in answering either of these questions. For these reasons, the present indications from PAMELA and FERMI have presented us with a unique opportunity of unraveling at least some of the mystery surrounding the dark matter. These experimental results, if they hold and get confirmed as due to dark matter, would strongly modify the way dark matter was perceived in the scientific community. In conclusion, let us note that several new experiments are being planned to explore the dark matter, directly or indirectly, and more information about the nature of the dark matter will be revealed in due cause. 


\section{Acknowledgements}

The authors thank PAMELA Collaboration, ATIC Collaboration and FERMI-LAT Collaboration for giving us permission to reproduce their figures. The authors thank Diptiman Sen for a careful reading of this article and useful comments. CJ would like to thank Gary Mamon for illuminating discussions regarding the search for dark matter in elliptical galaxies and clusters. The authors thank A Iyer for bringing to their notice a reference. Finally, the authors thank the anonymous referee for suggestions and comments which have contributed in improving the article.

\section{References}

[1] V Rubin, Scientific American 248, 96 (1983)

[2] Y Sofue and V Rubin, Ann. Rev. Astron. Astrophys. 39, 137 (2001), arXiv:astro-ph/ 0010594

[2a] In the absence of dark matter, one would expect that the curves to fall off as we move towards the outer parts of the Galaxy.

[3] J Binney and S Tremaine, Galactic dynamics (Princeton University Press, London, 1987)

[4] A Dekel, F Stoehr, G A Mamon, T J Cox, G S Novak and J R Primack, Nature 437, 707 (2005)

[5] G Mamon, in the meeting on Mass unveiling the mass: Extracting and interpreting galaxy masses (Kingston, Canada, 2009)

[6] F Zwicky, Helv. Phys. Acta 6, 110 (1933)

J Binney and S D Tremaine, Galactic dynamics, 2nd edn (Princeton University Press, 2007)

[7] C L Bennett et al, Astrophys. J. 464, L1 (1996), arXiv:astro-ph/9601067

J R Bond, G Efstathiou and M Tegmark, Mon. Not. R. Astron. Soc. 291, L33 (1997), arXiv:astro-ph/9702100

[8] Supernova Cosmology Project Collaboration: M Kowalski et al, Astrophys. J. 686, 749 (2008), arXiv:0804.4142 [astro-ph]

[9] WMAP Collaboration: G Hinshaw et al, Astrophys. J. Suppl. 180, 225 (2009), arXiv: 0803.0732 [astro-ph]

[10] E Komatsu et al, arXiv:0803.0547v2 [astro-ph] (preprint)

[11] S Dodelson, Modern cosmology (Academic Press, Amsterdam, The Netherlands, 2003) V Sahni, Lect. Notes Phys. 653, 141 (2004), arXiv:astro-ph/0403324

T Padmanabhan, Phys. Rep. 380, 235 (2003), arXiv:hep-th/0212290

E J Copeland, M Sami and S Tsujikawa, Int. J. Mod. Phys. D15, 1753 (2006), arXiv: hep-th/0603057

[12] J F Navarro, C S Frenk and S D M White, Astrophys. J. 490, 493 (1997)

[12a] Sharp increase in the density at the centre.

[13] W J G de Blok, S S McGaugh, A Bosma and V C Rubin, Astrophys. J. 552, L23 (2001)

[14] M Milgrom, Astrophys. J. 270, 365 (1983)

[15] O Tiret and F Combes, Astron. Astrophys. 464, 2, 517 (2007), arXiv:astro-ph/0701011

[16] $\mathrm{F}$ Combes and $\mathrm{O}$ Tiret, Invited paper presented at The Invisible Universe International Conference edited by J-M Alimi, A Fuzfa and P-S Corasaniti (AIP Publications), arXiv: 0908.3289 [astro-ph.CO]

[17] D Clowe, M Bradac, A H Gonzalez, M Markevitch, S W Randall, C Jones and D Zaritsky, Astrophys. J. 648, L109 (2006), arXiv:astro-ph/0608407 


\section{Results from PAMELA, ATIC and FERMI: Pulsars or dark matter?}

[17a] This is exactly analogous to the reason why the atomic hydrogen gas from two colliding galaxies is left at the centre of mass while the stars and the molecular gas pass through each other unaffected, as proposed and studied by Valluri et al [18] to explain the observed HI deficiency but normal molecular gas content of galaxies in clusters.

[17b] The relativistic MOND theory [19] proposed by Bekenstein could be used to explain the Bullet Cluster [20].

[18] M Valluri and C J Jog, Astrophys. J. 357, 367 (1990)

[19] J D Bekenstein, Phys. Rev. D70, 083509 (2004); Erratum, ibid. D71, 069901 (2005), arXiv:astro-ph/0403694

[20] J D Bekenstein, Nucl. Phys. A827, 555C (2009), arXiv:0901.1524 [astro-ph]

[21] V Trimble, Ann. Rev. Astron. Astrophys. 25, 425 (1987)

[22] G D’Amico, M Kamionkowski and K Sigurdson, arXiv:0907.1912 [astro-ph] (2009)

[23] D Pfenniger, F Combes and L Martinet, Astron. Astrophys. 285, 79 (1994)

[24] E Witten, Phys. Rev. D30, 272 (1984)

[25] J e Alam, S Raha and B Sinha, Astrophys. J. 513, 572 (1999), arXiv:astro-ph/9704226

[26] For an earlier discussion on this topic, see, A Bhattacharyya, J e Alam, S Sarkar, P Roy, B Sinha, S Raha and P Bhattacharjee, Nucl. Phys. A661, 629 (1999), arXiv:hep-ph/ 9907262, and references therein

[27] S Banerjee, S K Ghosh, S Raha and D Syam, Phys. Rev. Lett. 85, 1384 (2000), arXiv: hep-ph/0006286

J E Horvath, Astrophys. Space Sci. 315, 361 (2008), arXiv:0803.1795 [astro-ph]

For a recent summary on this topic, see S K Ghosh, arXiv:0808.1652 [astro-ph]

[28] T Schwetz, M A Tortola and J W F Valle, New J. Phys. 10, 113011 (2008), arXiv:0808.2016 [hep-ph]

[28a] Depending on the mass of the particle which sets its thermal and relativistic properties, dark matter can be classified as hot, warm and cold [29].

[29] J A Peacock, Cosmological physics (Cambridge University Press, Cambridge, 1999)

[30] A Strumia and F Vissani, arXiv:hep-ph/0606054

[31] M Viel, J Lesgourgues, M G Haehnelt, S Matarrese and A Riotto, Phys. Rev. Lett. 97, 071301 (2006), arXiv:astro-ph/0605706

[32] J Lesgourgues and S Pastor, Phys. Rep. 429, 307 (2006), arXiv:astro-ph/0603494

[33] A Palazzo, D Cumberbatch, A Slosar and J Silk, Phys. Rev. D76, 103511 (2007), arXiv: 0707.1495 [astro-ph]

[33a] On the other hand, if the neutrinos are not thermally produced and their production is suppressed as in models with low reheating temperature [34], it is possible to weaken the cosmological bounds, especially from extra galactic radiation and distortion of CMBR spectra [35] (see also [36]).

[34] G Gelmini, S Palomares-Ruiz and S Pascoli, Phys. Rev. Lett. 93, 081302 (2004), arXiv:astro-ph/0403323

[35] G Gelmini, E Osoba, S Palomares-Ruiz and S Pascoli, J. Cosmol. Astropart. Phys. 810, 29 (2008), arXiv:0803.2735 [astro-ph]

[36] M A Acero and J Lesgourgues, Phys. Rev. D79, 045026 (2009), arXiv:0812.2249 [astro-ph]

[37] C Boehm and P Fayet, Nucl. Phys. B683, 219 (2004), arXiv:hep-ph/0305261

[38] P Fayet, Phys. Rev. D75, 115017 (2007), arXiv:hep-ph/0702176

[39] S Gopalakrishna, S J Lee and J D Wells, Phys. Lett. B680, 88 (2009), arXiv:0904.2007 [hep-ph]

[40] N Borodatchenkova, D Choudhury and M Drees, Phys. Rev. Lett. 96, 141802 (2006), arXiv:hep-ph/0510147

[41] R Barbieri and L J Hall, arXiv:hep-ph/0510243

Pramana - J. Phys., Vol. 76, No. 1, January 2011 


\section{Debtosh Chowdhury, Sudhir K Vempati and Chanda J Jog}

[42] Q H Cao, E Ma and G Rajasekaran, Phys. Rev. D76, 095011 (2007), arXiv:0708.2939 [hep-ph]

[43] R Barbieri, L J Hall and V S Rychkov, Phys. Rev. D74, 015007 (2006), arXiv:hep-ph/ 0603188

[44] R D Peccei and H R Quinn, Phys. Rev. Lett. 38, 1440 (1977)

[45] R D Peccei, Lect. Notes Phys. 741, 3 (2008), arXiv:hep-ph/0607268

[46] G Bertone, D Hooper and J Silk, Phys. Rep. 405, 279 (2005), arXiv:hep-ph/0404175

[47] S P Martin, A Supersymmetry Primer, arXiv:hep-ph/9709356

[48] M Drees, R Godbole and P Roy, Theory and phenomenology of sparticles: An account of four-dimensional $\mathcal{N}=1$ supersymmetry in high energy physics (World Scientific, Hackensack, USA, 2004)

[48a] The corresponding symmetry here is called $R$-parity. If this symmetry is exact, the particle is stable. If it is broken mildly, the LSP could be sufficiently long-lived, close to the age of the Universe.

[49] G Jungman, M Kamionkowski and K Griest, Phys. Rep. 267, 195 (1996)

[49a] The neutralino could be either gaugino-dominated or higgsino-dominated depending on the composition. It turns out that neutralino composition should be sufficiently well-tempered [50] to explain the observed relic density. While one might debate the somewhat philosophical requirement of 'fine-tuning', it is now known that in the simplest models of supersymmetry breaking, like mSUGRA, only special regions in the parameter space, corresponding to the special conditions in the neutralino-neutralino annihilation channels satisfy the relic density constraint [51].

[50] N Arkani-Hamed, A Delgado and G F Giudice, Nucl. Phys. B741, 108 (2006), arXiv: hep-ph/0601041

[51] A Djouadi, M Drees and J L Kneur, J. High Energy Phys. 0603, 033 (2006), arXiv: hep-ph/0602001

[52] See for example, L Covi and J E Kim, New J. Phys. 11, 105003 (2009), arXiv:0902.0769 [astro-ph.CO] and references therein

[53] N Arkani-Hamed, S Dimopoulos and G R Dvali, Phys. Lett. B429, 263 (1998), arXiv: hep-ph/9803315

[54] N Arkani-Hamed, S Dimopoulos and G R Dvali, Phys. Rev. D59, 086004 (1999), arXiv: hep-ph/9807344

[54a] The extra space dimensions are compactified. The compact extra dimension manifests itself in ordinary four-dimensional space-time as an infinite tower of massive particles called Kaluza-Klein (KK) particles.

[55] L Randall and R Sundrum, Phys. Rev. Lett. 83, 3370 (1999), arXiv:hep-ph/9905221

[56] L Randall and R Sundrum, Phys. Rev. Lett. 83, 4690 (1999), arXiv:hep-th/9906064

[57] G Servant and T M P Tait, Nucl. Phys. B650, 391 (2003), arXiv:hep-ph/0206071

[58] D Hooper and S Profumo, Phys. Rep. 453, 29 (2007), arXiv:hep-ph/0701197

[59] H C Cheng, J L Feng and K T Matchev, Phys. Rev. Lett. 89, 211301 (2002)

[60] G Bertone, G Servant and G Sigl, Phys. Rev. D68, 044008 (2003), arXiv:hep-ph/0211342

[61] J Hubisz and P Meade, Phys. Rev. D71, 035016 (2005), arXiv:hep-ph/0411264

[62] G Belanger, A Pukhov and G Servant, J. Cosmol. Astropart. Phys. 0801, 009 (2008), arXiv:0706.0526 [hep-ph]

[63] I F M Albuquerque, L Hui and E W Kolb, Phys. Rev. D64, 083504 (2001), arXiv:hep-ph/ 0009017

[64] D J H Chung, E W Kolb and A Riotto, Phys. Rev. Lett. 81, 4048 (1998), arXiv: hep-ph/9805473

E W Kolb, D J H Chung and A Riotto, arXiv:hep-ph/9810361 
Results from PAMELA, ATIC and FERMI: Pulsars or dark matter?

[65] I F M Albuquerque and C Perez de los Heros, Phys. Rev. D81, 063510 (2010), arXiv: 1001.1381 [astro-ph.HE]

[65a] It cannot have electromagnetic interactions as this would mean it is charged, and it cannot have strong interactions as this would most likely mean it would be baryonic in from. Both these prospects are already ruled out by experiments.

[65b] In spite of being electrically neutral, dark-matter particle can have a non-zero electric and/or magnetic dipole moment, if it has a non-zero spin. In such a case the strongest constraint comes from Big Bang Nucleosynthesis. Interested readers are referred to the paper by Kamoinkowski et al [66] and particularly figure 1 therein.

[65c] More generally, the 'WIMP-nucleon cross-section' can be divided into (i) elastic spindependent (eSD), (ii) elastic spin-independent (eSI), (iii) inelastic spin-dependent (iSD) and (iv) inelastic spin-independent (iSI)

[66] K Sigurdson, M Doran, A Kurylov, R R Caldwell and M Kamionkowski, Phys. Rev. D70, 083501 (2004), Erratum, ibid. D73, 089903 (2006), arXiv:astro-ph/0406355

[66a] These are typically the same experiments which measure the cosmic ray spectrum. For a comprehensive list of all these experiments and other useful information like propagation packages, see http://www.mpi-hd.mpg.de/hfm/CosmicRay/CosmicRaySites.html.

[67] P Bhattacharjee and G Sigl, Phys. Rep. 327, 109 (2000), arXiv:astro-ph/9811011

[68] For a discussion of this effect, please see D Hooper, arXiv:0901.4090 [hep-ph]

[69] P Jean et al, Astron. Astrophys. 407, L55 (2003), arXiv:astro-ph/0309484

[69a] It should be noted that the integral spectrometer has a very good resolution of about $2 \mathrm{keV}$ over a range of energies $20 \mathrm{keV}$ to $8 \mathrm{MeV}$.

[70] G Weidenspointner et al, Nature 451, 159 (2008)

[71] DAMA Collaboration: R Bernabei et al, Eur. Phys. J. C56, 333 (2008), arXiv:0804.2741 [astro-ph]

[71a] Looking for such modulations further limit any systematics present in the experiment.

[71b] These results have been recently updated with six annual cycles for DAMA/LIBRA; the CL has now moved up to $8.9 \sigma$ [72].

[72] R Bernabei et al, Eur. Phys. J. C67, 39 (2010), arXiv:1002.1028 [astro-ph.GA]

[72a] The final results have a non-zero probability of two events in the signal region, we comment on it in the next section.

[73] XENON Collaboration: J Angle et al, Phys. Rev. Lett. 100, 021303 (2008), arXiv:0706. 0039 [astro-ph]

[74] XENON100 Collaboration: E Aprile et al, Phys. Rev. Lett. 105, 131302 (2010), arXiv: 1005.0380 [astro-ph.CO]

[75] CDMS Collaboration: Z Ahmed et al, Phys. Rev. Lett. 102, 011301 (2009), arXiv:0802. 3530 [astro-ph]

[76] CoGeNT Collaboration: C E Aalseth et al, Phys. Rev. Lett. 101, 251301 (2008); Erratum, ibid. 102, 109903 (2009), arXiv:0807.0879 [astro-ph]

[77] CoGeNT Collaboration: C E Aalseth et al, arXiv:1002.4703 [astro-ph.CO]

[78] S Chang, J Liu, A Pierce, N Weiner and I Yavin, J. Cosmol. Astropart. Phys. 1008, 018 (2010), arXiv:1004.0697 [hep-ph]

[79] J Kopp, T Schwetz and J Zupan, J. Cosmol. Astropart. Phys. 1002, 014 (2010), arXiv:0912.4264 [hep-ph]

[80] PAMELA Collaboration: O Adriani et al, Nature 458, 607 (2009), arXiv:0810.4995 [astro-ph]

[81] J Chang et al, Nature 456, 362 (2008)

[82] HESS Collaboration: F Aharonian et al, Phys. Rev. Lett. 101, 261104 (2008), arXiv:0811. 3894 [astro-ph] 


\section{Debtosh Chowdhury, Sudhir K Vempati and Chanda J Jog}

[83] The Fermi LAT Collaboration: A A Abdo et al, Phys. Rev. Lett. 102, 181101 (2009), arXiv:0905.0025 [astro-ph.HE]

[84] I V Moskalenko and A W Strong, Astrophys. J. 493, 694 (1998)

[85] A W Strong and I V Moskalenko, Adv. Space Res. 27, 717 (2001), arXiv:astro-ph/0101068

[86] AMS Collaboration: M Aguilar et al, Phys. Rep. 366, 331 (2002); Erratum, ibid. 380, 97 (2003)

[87] S W Barwick et al, Astrophys. J. 498, 779 (1998), arXiv:astro-ph/9712324

[88] S Torii et al, Astrophys. J. 559, 973 (2001)

[89] PPB-BETS Collaboration: S Torii et al, arXiv:0809.0760 [astro-ph]

[90] A W Strong, I V Moskalenko and O Reimer, Astrophys. J. 613, 962 (2004), arXiv: astro-ph/0406254

[91] http://galprop.stanford.edu/web_galprop/galprop_home.html

[92] http://www-ekp.physik.uni-karlsruhe.de/ zhukov/GalProp/galpropanal.html

[93] http://apcauger.in2p3.fr/CRPropa/index.php

[93a] Some codes are constructed to fix the various parameters of their own cosmic ray propagation model (see for example, DRAGON [94]). Here one can fix the diffusion coefficients from PAMELA and other experimental data.

[94] C Evoli, D Gaggero, D Grasso and L Maccione, J. Cosmol. Astropart. Phys. 0810, 018 (2008), arXiv:0807.4730 [astro-ph]

[95] http://crt.osu.edu/

[96] http://wwwasd.web.cern.ch/wwwasd/geant/

[97] L Desorgher, E O Fluckiger, M R Moser and R Butikofer, Prepared for 28th International Cosmic Ray Conferences (ICRC 2003) (Tsukuba, Japan, 31 Jul-7 Aug 2003)

[98] See for example, A Strumia, Talk presented at Planck 2009, http://www.pd.infn.it/ planck09/Talks/Strumia.pdf P Meade, M Papucci, A Strumia and T Volansky, arXiv:0905.0480 [hep-ph]

[99] P Gondolo, J Edsjo, P Ullio, L Bergstrom, M Schelke and E A Baltz, J. Cosmol. Astropart. Phys. 0407, 008 (2004), arXiv:astro-ph/0406204

[99a] For an independent analysis which confirms the PAMELA excess, see [100].

[100] T Delahaye, F Donato, N Fornengo, J Lavalle, R Lineros, P Salati and R Taillet, Astron. Astrophys. 501, 821 (2009), arXiv:0809.5268 [astro-ph]

[100a] The cosmic ray electrons follow a power-law spectrum, with an index $\sim-3$. Thus it is normalized by a factor $E^{3}$.

[101] D Hooper, P Blasi and P D Serpico, J. Cosmol. Astropart. Phys. 0901, 025 (2009), arXiv:0810.1527 [astro-ph]

[102] P Blasi, arXiv:0903.2794 [astro-ph.HE]

[102a] The curvature radiation arises due to relativistic, charged particles moving around curved magnetic field lines (see for details, Gil et al [103]).

[103] J Gil, Y Lyubarsky and G I Melikidze, Astrophys. J. 600, 872 (2004), arXiv:astro-ph/ 0310621

[104] S Coutu et al, Astropart. Phys. 11, 429 (1999)

[105] A M Atoian, F A Aharonian and H J Volk, Phys. Rev. D52, 3265 (1995)

[106] H Yuksel, M D Kistler and T Stanev, Phys. Rev. Lett. 103, 051101 (2009), arXiv:0810.2784 [astro-ph]

[107] I Büsching, O C de Jager, M S Potgieter and C Venter, Astrophys. J. 78, L39 (2008)

[108] M Ahlers, P Mertsch and S Sarkar, arXiv:0909.4060 [astro-ph.HE]

[109] P Mertsch and S Sarkar, arXiv:0905.3152 [astro-ph.HE]

[110] J Lavalle, Q Yuan, D Maurin and X J Bi, Astron. Astrophys. 479, 427 (2008), arXiv: 0709.3634 [astro-ph] 
Results from PAMELA, ATIC and FERMI: Pulsars or dark matter?

[111] J Hisano, S Matsumoto and M M Nojiri, Phys. Rev. Lett. 92, 031303 (2004), arXiv: hep-ph/0307216

J Hisano, S Matsumoto, M M Nojiri and O Saito, Phys. Rev. D71, 063528 (2005), arXiv:hep-ph/0412403

[112] For a recent discussion, see S Hannestad and T Tram, arXiv:1008.1511 [astro-ph.CO]

[112a] It should also be considered while computing annhilation cross-section in the early Universe $[112 \mathrm{c}]$.

[112b] S Mohanty, R Rao and D P Roy, Reconciling heavy wino dark matter model with the relic density and PAMELA data using Sommerfeld effect, arXiv:1009.5058 [hep-ph]

[112c] Non-thermal production typically refers to production mechanisms through decays of very heavy particles like inflaton [113].

[113] D J H Chung, E W Kolb and A Riotto, Phys. Rev. D60, 063504 (1999), arXiv:hep-ph/ 9809453

[114] S Dodelson, A V Belikov, D Hooper and P Serpico, Phys. Rev. D80, 083504 (2009), arXiv:0903.2829 [astro-ph.CO]

[115] A V Belikov and D Hooper, arXiv:0906.2251 [astro-ph.CO]

[116] I Cholis, G Dobler, D P Finkbeiner, L Goodenough, T R Slatyer and N Weiner, arXiv:0907.3953 [astro-ph.HE]

[117] D Hooper and K M Zurek, arXiv:0909.4163 [hep-ph]

[118] L Goodenough and D Hooper, arXiv:0910.2998 [hep-ph]

[119] M Pato, L Pieri and G Bertone, arXiv:0905.0372 [astro-ph.HE]

[120] N Arkani-Hamed, D P Finkbeiner, T R Slatyer and N Weiner, Phys. Rev. D79, 015014 (2009), arXiv:0810.0713 [hep-ph]

[121] A Katz and R Sundrum, J. High Energy Phys. 0906, 003 (2009), arXiv:0902.3271 [hep-ph]

[122] I Cholis, L Goodenough and N Weiner, Phys. Rev. D79, 123505 (2009), arXiv:0802.2922 [astro-ph]

[123] I Cholis, L Goodenough, D Hooper, M Simet and N Weiner, arXiv:0809.1683 [hep-ph]

[124] I Cholis, D P Finkbeiner, L Goodenough and N Weiner, arXiv:0810.5344 [astro-ph]

[125] D Hooper and T M P Tait, Phys. Rev. D80, 055028 (2009), arXiv:0906.0362 [hep-ph]

[126] I Gogoladze, N Okada and Q Shafi, Phys. Lett. B679, 237 (2009), arXiv:0904.2201 [hep-ph]

[127] D Hooper and K M Zurek, Phys. Rev. D79, 103529 (2009), arXiv:0902.0593 [hep-ph]

[127a] Some of the first simulations using PYTHIA and DARK SUSY for the KK gravition can be found in [128]. Similar study for SUSY can be found in [129]. These have been done when HEAT results have shown an excess though in a less statistically significant way.

[128] D Hooper and G D Kribs, Phys. Rev. D70, 115004 (2004), arXiv:hep-ph/0406026

[129] E A Baltz and J Edsjo, Phys. Rev. D59, 023511 (1998), arXiv:astro-ph/9808243

[130] M Ibe, H Murayama, S Shirai and T T Yanagida, J. High Energy Phys. 0911, 120 (2009), arXiv:0908.3530 [hep-ph]

[131] A Ibarra, D Tran and C Weniger, arXiv:0906.1571 [hep-ph]

[132] A Ibarra, D Tran and C Weniger, arXiv:0909.3514 [hep-ph]

[133] A Ibarra and D Tran, J. Cosmol. Astropart. Phys. 0902, 021 (2009), arXiv:0811.1555 [hep-ph]

[134] E Nardi, F Sannino and A Strumia, J. Cosmol. Astropart. Phys. 0901, 043 (2009), arXiv:0811.4153 [hep-ph]

[135] W Buchmuller, L Covi, K Hamaguchi, A Ibarra and T Yanagida, J. High Energy Phys. 0703, 037 (2007), arXiv:hep-ph/0702184

[136] I Gogoladze, R Khalid, Q Shafi and H Yuksel, Phys. Rev. D79, 055019 (2009), arXiv:0901.0923 [hep-ph] 
[137] W Buchmuller, A Ibarra, T Shindou, F Takayama and D Tran, J. Cosmol. Astropart. Phys. 0909, 021 (2009), arXiv:0906.1187 [hep-ph]

[138] A Arvanitaki, S Dimopoulos, S Dubovsky, P W Graham, R Harnik and S Rajendran, Phys. Rev. D79, 105022 (2009), arXiv:0812.2075 [hep-ph]

[139] A Arvanitaki, S Dimopoulos, S Dubovsky, P W Graham, R Harnik and S Rajendran, Phys. Rev. D80, 055011 (2009), arXiv:0904.2789 [hep-ph]

[140] M R Buckley, K Freese, D Hooper, D Spolyar and H Murayama, arXiv:0907.2385 [astro-ph.HE]

[141] M Fairbairn and J Zupan, arXiv:0810.4147 [hep-ph]

[142] D Malyshev, I Cholis and J Gelfand, Phys. Rev. D80, 063005 (2009), arXiv:0903.1310 [astro-ph.HE]

[143] FERMI-LAT Collaboration: D Grasso et al, arXiv:0905.0636 [astro-ph.HE]

[144] M Kadastik, M Raidal and A Strumia, arXiv:0908.1578 [hep-ph]

[145] J Hisano, M Kawasaki, K Kohri and K Nakayama, Phys. Rev. D79, 043516 (2009), arXiv:0812.0219 [hep-ph]

[146] J Goodman, M Ibe, A Rajaraman, W Shepherd, T M P Tait and H B P Yu, arXiv:1005.1286 [hep-ph]

J Goodman, M Ibe, A Rajaraman, W Shepherd, T M P Tait and H B P Yu, arXiv:1008.1783 [hep-ph]

[147] The CDMS-II Collaboration: Z Ahmed et al, arXiv:0912.3592 [astro-ph.CO] 